\title{
Closed-Loop Feedback Illumination for Optical Inverse Tone-Mapping in Light Microscopy
}

\author{
Oliver Bimber , Daniel Klöck , Toshiyuki Amano , Anselm Grundhöfer, Daniel Kurz
}

\begin{abstract}
In this article, we show that optical inverse tone-mapping (OITM) in light microscopy can improve the visibility of specimens, both when observed directly through the oculars and when imaged with a camera. In contrast to previous microscopy techniques, we pre-modulate the illumination based on the local modulation properties of the specimen itself. We explain how the modulation of uniform white light by a specimen can be estimated in real time, even though the specimen is continuously but not uniformly illuminated. This information is processed and back-projected constantly, allowing the illumination to be adjusted on the fly if the specimen is moved or the focus or magnification of the microscope is changed. The contrast of the specimen's optical image can be enhanced, and highintensity highlights can be suppressed. A formal pilot study with users indicates that this optimizes the visibility of spatial structures when observed through the oculars. We also demonstrate that the signal-to-noise $(\mathrm{S} / \mathrm{N})$ ratio in digital images of the specimen is higher if captured under an optimized rather than a uniform illumination. In contrast to advanced scanning techniques that maximize the $\mathrm{S} / \mathrm{N}$ ratio using multiple measurements, our approach is fast because it requires only two images. This can improve image analysis in digital microscopy applications with real-time capturing requirements.
\end{abstract}

Index Terms-Computer graphics, picture/image generation, display algorithms, image processing, enhancement.

\section{INTRODUCTION AND MOTIVATION}

In light microscopy (or optical microscopy), visible light is either transmitted through or reflected from a specimen before it is observed or recorded. In bright field microscopy, the illumination light is modulated in intensity and color depending on the specimen's transmission or reflection properties before it enters the objective lens. The resolution of this method is limited by the contrast and the wavelength of visible light. Several other imaging and illumination techniques are commonly employed in light microscopes to enhance contrast, such as dark field, phase contrast, (differential) interference contrast, fluorescence, oblique, and Rheinberg illumination. By exploiting refraction, diffraction, interference, or fluorescence of light, these methods are applied mainly to make objects, for instance biological structures such as cells, visible that are otherwise invisible. Compared to bright field microscopy, the optical images produced with such contrast techniques usually give an unnatural appearance to the observed specimens. A considerable degree of experience is required to interpret correctly the resulting gradient and false color images. In addition, specimens that are too thick for transmitted light require a reflected illumination, for which only a few means of contrast enhancement are currently available. Since a detailed discussion of these techniques is beyond the scope of this article, interested readers are referred

Bimber is with the Johannes Kepler University Linz, Austria, E-Mail: oliverbimber@jku.at

Klöck is with the Brandenburg Technical University Cottbus, Germany, EMail: dkloeck@informatik.tu-cottbus.de

Amano is with the Nara Institute of Science and Technology, Japan, E-Mail: amano@is.naist.jp

Grundhöfer and Kurz are with the Bauhaus-University Weimar, Germany, E-Mail: \{grundhoe,kurz\}@uni-weimar.de to microscopy text books, such as [16], and to online sources ${ }^{1}$. In this paper, we focus entirely on enhancing classical bright field microscopy.

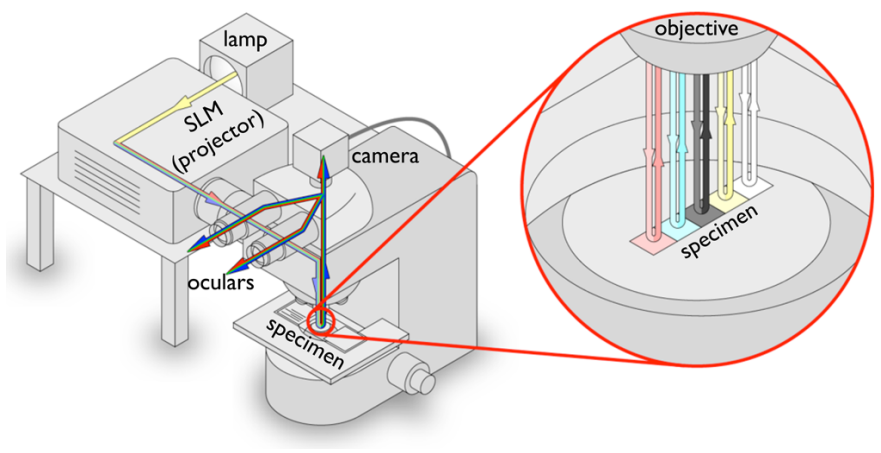

Fig. 1. Schematic drawing of optical inverse tonemapping in bright field microscopy: The illumination is pre-modulated by the spatial light modulator (SLM) of a projector, based on the camera-recorded local modulation properties of the specimen itself. It is modulated a second time when transmitted or reflected by a specimen. This results in a contrast-enhanced optical image that can be recorded with a camera or observed through the oculars. The drawing illustrates an example of reflected illumination. Prototypes for reflected and transmitted illumination are presented in section 5 .

In domains that utilize bright field microscopy, such as microscopic surgery, forensic analysis, materials science, and bioimaging, contrast is often too low or too high, making observations directly through the oculars

1. http://www.microscopyu.com/,http://www.olympusmicro.com/, http://zeiss-campus.magnet.fsu.edu/ 
problematic, since the capabilities of the human visual system are limited. Low contrast can result from lowcontrast specimens or a high degree of scattering within the specimen. Excessively high contrast can be the result of specular highlights on the specimen. Furthermore, the acquisition of extremely low- or high-contrast images is problematic in many digital microscopy applications that rely on robust image analysis.

In this article, we describe how the visibility of specimens observed through the oculars can be enhanced, and how the signal-to-noise $(\mathrm{S} / \mathrm{N})$ ratio of captured images can be increased using a technique we refer to as optical inverse tone-mapping (OITM). In both cases, we control the microscope illumination spatially in real time, as illustrated in figure 1. This allows highly interactive and dynamic optical examinations and image analysis tasks that involve specimen movements and changing observation parameters, such as focus and magnification. This is essential in many applications of light microscopy and related domains, including endoscopy and microscopic surgery.

Unlike the contrast techniques listed above, our approach allows visibility enhancement while preserving the natural appearance of the specimens. It holds particular potential for real-time applications that depend on bright field microscopy and require a reflected illumination.

\section{Related Work and Contribution}

Spatial Light Modulation in Optical Microscopy. Spatial light modulators (SLMs), such as LCDs and DMDs, are used at different stages of the microscope optical train to generate or enhance contrast. Figure 2 complements the following summary with an overview of a simplified microscope optical train.

SLMs are integrated at the objective back focal plane to apply phase-stepping contrast techniques [22] or point-spread-function shaping to reduce aberrations in confocal microscopy [20]. They generate annulus masks dynamically at the condenser aperture plane to support imaging and illumination techniques, such as dark-field, Rheinberg, and oblique illumination, but also optical staining without the need to change physical filters mechanically [26]. SLMs are also, as in our case, focused at the field aperture plane. In fluorescence microscopy, for instance, this allows different areas of the specimen to be excited individually by using manually defined binary illumination patterns [28], [9]. This method avoids high scattering and color artifacts that are caused by autofluorescence. In addition, dynamic field apertures (instead of static ones) are also employed in modern ophthalmological devices for examining the eyeballs and for protecting the retina from bright light during eye surgery. Light field microscopy [13] captures $4 \mathrm{D}$ radiant light fields by placing a microlens array at the intermediate image plane of the imaging path. The incident light field can be controlled by using a second

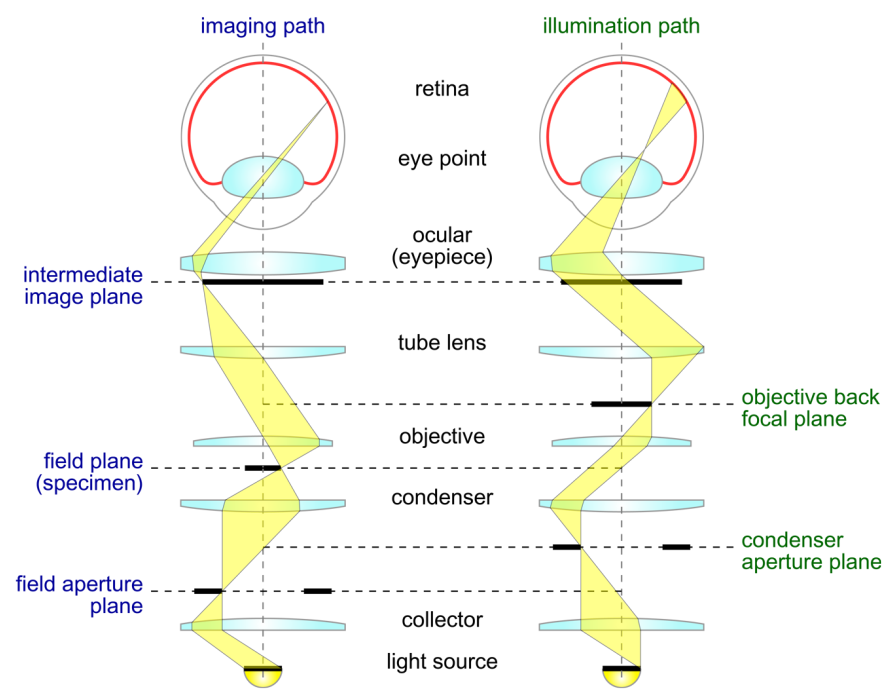

Fig. 2. Diagram of simplified microscope optical train: The condenser focuses the field aperture into the field plane and into the intermediate image plane (imaging path) and, at the same time, focuses the light sources into the condenser aperture plane and into the objective back focal plane (illumination path). For simplicity, the example shows a transmitted illumination configuration.

microlens array at the intermediate image plane of the illumination path and a projector [14]. Controlling the incident light field makes possible reproducing techniques such as darkfield and oblique illumination, and also allows directing and focusing light rays at selected positions and depths. Using a mechanism similar to that described in [9] this can also reduce negative effects caused by autofluorescence.

None of the techniques described above pre-modulate the illumination in intensity or color based on the local modulation properties of the specimen itself. We, however, show that this can improve the visibility of the resulting optical image when observed through the oculars or captured in digital images. Microscopy techniques that apply SLMs for scanning, measuring, superresolution imaging, and the like already exist. We enhance optical images in real time, rather than produce enhanced digital images using a substantial number of measurements.

High Dynamic Range Imaging and Optical ToneMapping. High dynamic range (HDR) imaging is frequently applied in microscopy. Like conventional multi-exposure techniques [8], alternative HDR imaging methods pre-attenuate the scene radiance on a per-pixel basis with SLMs before it reaches the camera sensor. In [15], an LCoS panel is used for pre-modulation. Multiple images are recorded while adapting the response of the LCoS panel iteratively until no sensor pixel remains saturated. Using the final camera image and attenuation values, the original scene radiance can be reconstructed. For optical tone-mapping, SLMs are 


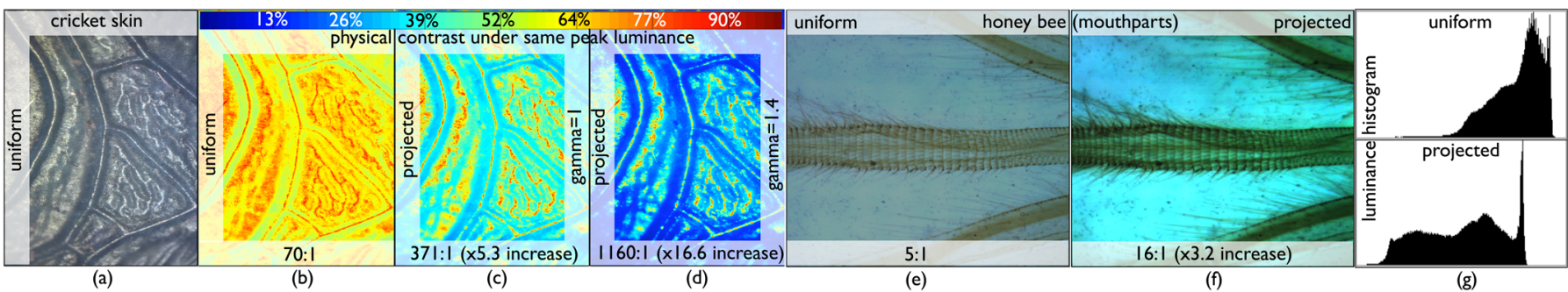

Fig. 3. Stretching physical contrast by optical inverse tone-mapping: A cricket skin captured under reflected illumination (a). The heat-maps illustrate the measured physical contrast under the same peak-luminance for uniform illumination (b) and for different contrast-stretched back-projected illuminations (c,d). The honey bee was captured under transmitted illumination: uniform white (e) and contrast-stretched back-projected (f). The histogram profiles are illustrated for both cases $(\mathrm{g})$. All measurements were taken from captured HDR images. The numbers indicate the contrast measured and the contrast increase relative to a uniform illumination.

also employed to attenuate the incoming scene radiance to the dynamic range of the camera sensor [17], [18], [11]. Some approaches need multiple recording iterations [11], while others support video-rate capturing [17]. However, such techniques normally do not produce high dynamic range images. Rather than a contrast enhancement, a contrast reduction of the scene radiance is usually achieved, and thus unsaturated low dynamicrange (LDR) images are captured. Optical tone-mapping is also used in optical see-through devices rather than in imaging applications [29]. In this case, contrast enhancement of the scene radiance is possible.

A fundamental limitation of HDR imaging and most optical tone-mapping techniques is that the contrast of the recorded or observed optical images themselves cannot be increased before being imaged or viewed. Such methods ensure only that the entire contrast range of the scene is captured or attenuated to the dynamic range of the camera. Although digital contrast techniques, such as inverse tone-mapping [5], could be applied after recording, imaging techniques alone cannot counter contrast reductions that result from light modulation by the specimen. Furthermore, details within high-intensity reflection regions also cannot be revealed after imaging under a uniform illumination. This also holds true for the optical see-through tonemapping technique in [29], since contrast enhancement or highlight suppression is performed after the light is modulated with the scene. Overcoming these problems is only possible if the illumination can be controlled before it is modulated by a scene or, as in our context, by a specimen. For the recording of high dynamic range radiance maps in microscopy, this was explored in [1], where multiple images were captured at the same exposure but with successively decreasing local illumination in saturated regions. However, another essential limitation of HDR imaging and optical tonemapping is that most techniques require capturing multiple images. This prevents them from recording dynamic scenes (e.g., moving specimens) or scenes under changing imaging conditions (e.g., adapting magnification and focus). This limitation also applies to programmable array microscopy [28], where the illumination path and the imaging path can be spatially modulated simultaneously.

Our technique, however, allows observing and recording contrast-enhanced optical images of specimens in real time and in interactive and dynamic situations. Low contrast that results from the modulation of light by the specimen can be amplified, since the irradiant illumination rather than the imaged radiance is modulated. Furthermore, highlighting relevant structures and suppressing the background leads to a decreased amount of scattering and hence to an additional gain in contrast. In addition, appropriate illumination control makes it possible to image and observe instantly details in regions of surface- and subsurface-reflections.

Projector-Based Illumination. Projectors have been used for spatial light modulation in backlights of high dynamic range displays that employ LCDs as front modulators [27]. Here, light is modulated twice; once by the backlight projector and a second time by the LCD front panel. This is generally referred to as double-modulation. It leads to high contrast and high dynamic range images if both modulators can be controlled with respect to the displayed content. A variety of other approaches exist that realize HDR displays based on this principle by applying different kinds of SLM, such as Liquid Crystal Displays (LCDs), Digital Micromirror Devices (DMDs), Light-Emitting Diodes (LEDs), and Liquid Crystal on Silicon (LCoS). Several projector-camera techniques exist that improve the quality of video presentations projected onto nonoptimized screens. They aim to compensate for artifacts that are caused by textured, colored, or non-planar surfaces. Some of them are related to our approach. In [24], for instance, displaying overlapping images from different angles using multiple projectors compensates for highlights on specular screen surfaces. The closedloop photometric adaptation method described in [10] is another example, which estimates the optimal compensation image to be projected onto a textured 
surface. It does not imply a static registration between projector and surface.

The techniques described in [6], [4] are closely related, since they also double-modulate pre-captured images on physical surfaces. In these cases, video projectors are registered precisely to paper printouts. By computing accurately the printed and the projected images, the method in [6], for instance, allows displaying high dynamic range images, such as those required for radiological visualizations. These were the first applications of OITM that used a projector-based illumination for contrast enhancement.

Summary of Contributions. In addition to applying OITM to microscopy, we present three essential contributions: Firstly, we introduce a closed-loop feedback framework that allows OITM to be applied in real time, thus enabling interactive and dynamic optical examinations and image analysis tasks. We explain the implications of OITM in the context of complex light modulation caused by microscopic specimens and present solutions that make interactive applications of OITM more robust. Secondly, we demonstrate that the $\mathrm{S} / \mathrm{N}$ ratio in images that are optically contrast-enhanced with OITM before recording is higher than in images that are captured under uniform illumination. In contrast to advanced scanning or smoothing techniques that maximize the $\mathrm{S} / \mathrm{N}$ ratio by means of multiple measurements, our approach is fast because it requires only two images. Thirdly, we present a formal pilot study with users, which indicates that OITM outperforms a uniform illumination in directly perceiving small and large specimen details through the oculars.

\section{Optical InVERSE TONE-MAPPING}

\subsection{Basic Principle}

To enhance contrast with OITM, we can capture the specimen under uniform (white) illumination, contraststretch the recorded image, and then project the result back to the specimen, as illustrated in figure 1. Thus, contrast amplification of the optical image is achieved by double-modulation: The back-projected image of the specimen is modulated a second time by the specimen itself. In light microscopy, this is feasible under both transmitted and reflected illumination. Prototype configurations for these cases are presented in section 5 . Figure 3 shows two specimens for which the physical contrast was boosted optically with OITM. We achieved increases in physical contrast of up to a factor of 16 (e.g., for the cricket skin shown in figure 3d) and up to 11,000:1 (e.g., when contrast-boosting the pyrite sample shown in figure 10).

The behavior of OITM can be explained using the principles of light transport. As illustrated in figure 4, we assume that camera and projector optics are perfectly co-aligned in the microscope optical train (sym-

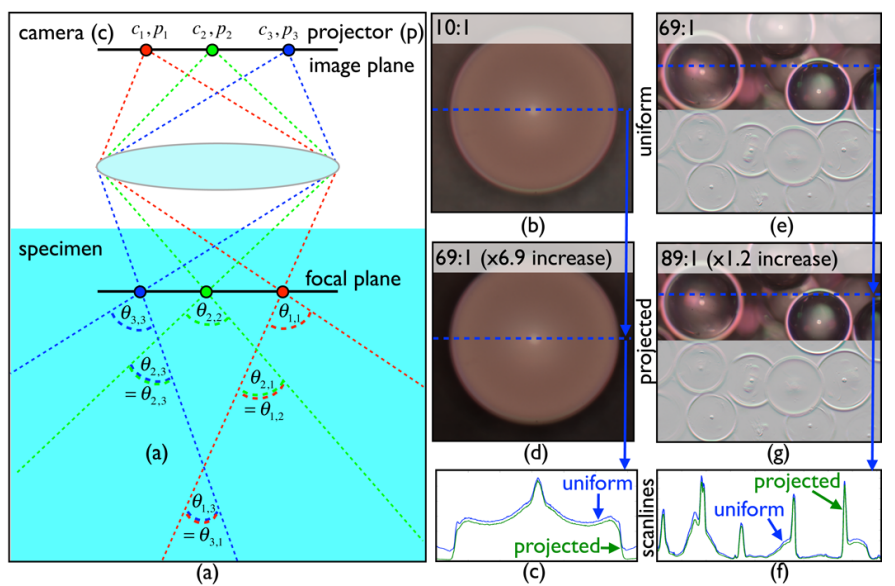

Fig. 4. OITM light transport: Co-aligned projector-camera pixels are focused on a plane inside a specimen volume (a). Light can be transported from all projector pixels through the volume (where it interacts) to all camera pixels (where it is integrated). If the interaction of light is dominant (e.g., due to a high degree of scattering), then OITM becomes inefficient but does not geometrically bias the resulting optical image. This is shown using transparent $500-600 \mu \mathrm{m}$ thick polystyrene microspheres that are illuminated under uniform white $(b, e)$ and under OITM (c,f) illumination. For a single bead on an opaque background, a contrast increase of a factor of 6.9 can be achieved $(b, c)$. For a $1 \mathrm{~cm}$ thick pile of beads, the contrast increase drops to a factor of 1.2. The reason for this is the significantly larger amount of scattering inside the volume of the bead pile. The gradient inlays and scanline plots in d-g illustrate that, in both cases, features and their gradient profiles are preserved, while only gradient magnitudes vary due to contrast enhancement.

bolically represented by a single lens). In this case, each camera/projector-pixel pair on the shared image plane can both emit and integrate light rays (at the same optically folded solid angle) that intersect at the adjusted focal plane, but may continue in non-opaque specimen volumes. Light can be transported from each projector pixel $p$ to each camera pixel $c$ while being modulated on the way. This modulation can be described by the forward light transport, with $\theta_{i, j}$ representing the transport from $p_{i}$ to $c_{j}$ (given that Helmholtz reciprocity is valid, then $\left.\theta_{i, j}=\theta_{j, i}\right)$ :

$$
\left[\begin{array}{c}
c_{1} \\
\ldots \\
c_{n}
\end{array}\right]=\left[\begin{array}{ccc}
\theta_{1,1} & \ldots & \theta_{n, 1} \\
\ldots & \ldots & \ldots \\
\theta_{1, n} & \ldots & \theta_{n, n}
\end{array}\right]\left[\begin{array}{c}
p_{1} \\
\ldots \\
p_{n}
\end{array}\right]
$$

If $T$ denotes the symmetric light transport matrix and $\left[p_{1}, \ldots, p_{n}\right]=[1, \ldots, 1]=p$ describes a uniform illumination, then the resulting camera image $\left[c_{1}, \ldots, c_{n}\right]=c$ can be computed according to $c=T p=T 1$, with 1 indicating an all-ones vector. We can assume that the camera image is proportional to the optical image that can be observed through the oculars. If this image is back- 
projected with OITM, the new resulting camera image is $c^{\prime}=T c=T(T 1)=T^{2} 1$. This means that, in this case, the result of OITM is equivalent to squaring the entire light transport (with $T^{2}$ also being symmetric). For specimens that cause little scattering (e.g., thin or opaque specimens), $T$ is sparse and OITM is efficient, since $T^{2}$ enhances the contrast of the direct light transport (e.g., caused by direct reflection or transmission). When more scattering occurs (e.g., caused by specimens with a high degree of volume scattering or strong surface interreflections), then $T$ is denser, and OITM becomes less effective. Compared to a uniform illumination, OITM preserves gradient profiles of features and therefore does not geometrically bias the resulting optical image. If an additional contrast-stretching function $(f)$ is applied to $c$ before back-projecting it, then $T$ is not simply squared, but OITM results in a more complex light transport: $c^{\prime}=T f(c)=T f(T 1)$. Nonetheless, gradient profiles are preserved if $f$ is monotonically increasing. Two examples with different degrees of scattering are illustrated in figures $4(\mathrm{~b}-\mathrm{g})$.

\subsection{Increasing S/N Ratio}

Especially in photon-limited situations in which photon noise is the major source of noise (which applies to most cases in light microscopy), the signal-to-noise ratio can be increased by stretching the physical contrast optically with OITM before capturing images that are processed further. Depending on the specimen, we achieve a $\mathrm{S} / \mathrm{N}$ gain of up to a factor of 3-4 in our experiments with our current prototype. Figure 5 illustrates an example.

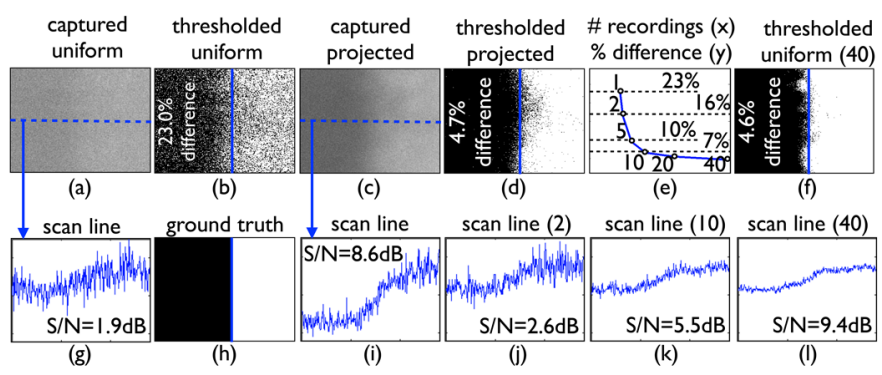

Fig. 5. Increasing $\mathrm{S} / \mathrm{N}$ ratio: Capturing a low-contrast edge (1.22:1) under white light (a) results in an image with a low $\mathrm{S} / \mathrm{N}$ ratio $(\mathrm{g})$. Contrast-stretching and backprojecting this image instead of using a uniform illumination enhances the signal through double-modulation with the specimen (c). A higher $\mathrm{S} / \mathrm{N}$ ratio (i) leads to improved results in digital image processing techniques, such as automatic thresholding [23]. Thresholding results (b), (d), and $(f)$ show the deviations from the ground truth $(h)$. Many images have to be recorded and averaged under uniform illumination for reducing noise at a constant signal level $(e, i-k)$ to achieve similar results $(f, l)$. The contrast edge was created on Kodak photo paper using a dyesublimation heat transfer printer.

We measured the $\mathrm{S} / \mathrm{N}$ ratio in each image with New- berry's analytical equation [21][16], which is frequently used in digital microscopy:

$$
S / N=\frac{\sqrt{C_{o}}}{\sqrt{\frac{1}{g}+\frac{n \sigma^{2}}{C_{o}}+\frac{n \sigma^{2}}{p C_{o}}}},
$$

where $1 d B=20 \log S / N, C_{o}=C_{f}-C_{b}$ is the object count in analog to digital units $(A D U)$ with $C_{f}$ and $C_{b}$ being the pixel sums within the selected regions that define the object fore- and background; $n$ is the number of pixels in the object area, $p$ is the number of pixels in the background area, $\sigma^{2}$ is the variance of background pixels (in $A D U^{2}$ ), and $g$ is the camera's electron gain in electrons/ADU (in 1/ADU).

Before back-projecting, we can also contrast-stretch the initial image $(M)$ recorded under white light, using nonlinear tone-mapping:

$$
L^{\prime}=\min \left(1, \max \left(0,\left(\frac{L-M I N-(M A X-M I N) o}{(M A X-M I N) w}\right)\right)\right)^{\gamma}
$$

where $L$ is the original luminance and $M A X-M I N$ is the original luminance range of $M$. A luminance window can be selected in this range by defining its offset $(o \in$ $[0,1])$ from $M I N$ and its width $(w \in] 0,1-o])$. It allows the relevant luminance range in $M$ to be extracted. Values within the window are first normalized to $[0,1]$ and are then re-mapped exponentially by $\gamma$. Thus, $w, o$, and $\gamma$ can be adjusted individually to achieve the highest possible contrast without causing saturation in local regions. The tone-mapped luminance $L^{\prime}$ is recombined with the chrominance in $M$ if a colored illumination is required. Otherwise, only $L$ is displayed in the dynamic range of the projector. Note that contrast-stretching $M$ boosts both the signal and the noise level in the illumination. Double-modulation with the specimen, however, amplifies the signal level more than the noise level, since the noise pattern is not present on the specimen. Furthermore, minimizing scattering (e.g., by the specimen) and defocus (e.g., due to the microscope's limited depth of field), and digital noise compensation reduce the highfrequency noise components in the illumination.

The $\mathrm{S} / \mathrm{N}$ gain for a sliced tissue sample and various exposure times is presented in figure 6 . Note that we used a Point Grey Dragonfly 2 CCD camera (37e/ADU) for these measurements to satisfy Nyquist's criterion for sampling the illumination pattern by the camera signal. In this case, the projector pixel size $(3.15 \mu \mathrm{m})$ is less than half the composite camera pixel size $(7.05 \mu \mathrm{m})$ on the field plane.

As shown in figure 6, the $\mathrm{S} / \mathrm{N}$ ratio in images that are optically contrast-enhanced before recording is always higher than in images that are captured under uniform illumination, even when employing additional digital contrast techniques. This also applies to HDR images computed from the individual exposures and to their tone-mapped LDR counterparts. For the example shown in figure 6, we measured an S/N gain of $27.9 d B / 13.6$ $d B=2.1$ in the resulting HDR images, and a gain of 

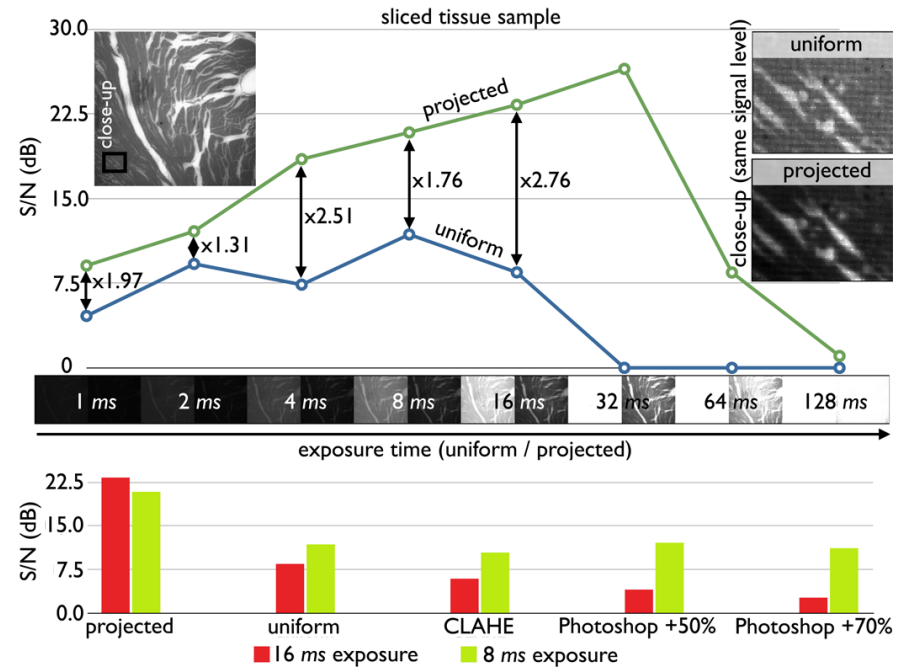

Fig. 6. $\mathrm{S} / \mathrm{N}$ ratio gain at different exposures (top): Comparing uniform white and contrast-stretched backprojected illumination with $\gamma=1.0$. For overexposed images $\mathrm{S} / \mathrm{N}$ is 0 . The close-ups visualize the $\mathrm{S} / \mathrm{N}$ ratio for uniform and projected illumination at the same signal level. Comparing optical contrast-enhancement before recording and digital contrast-enhancement after recording (bottom): For digital contrast-enhancement, we applied contrast limited histogram equalization (CLAHE) and Photoshop's non-linear contrast operator with two different contrast settings. Slight $\mathrm{S} / \mathrm{N}$ variations after digital contrast amplification are due to regional pixel saturations. The specimen is a sliced tissue sample.

$17.2 d B / 11.5 d B=1.5$ in linearly tone-mapped LDR versions that were computed from the corresponding HDR images.

An alternative to stretching the signal with OITM is smoothing the noise level by averaging multiple images recorded under uniform illumination. This increases the $\mathrm{S} / \mathrm{N}$ ratio by decreasing noise, while the signal remains at the same level, since contrast is not enhanced. For lowcontrast specimens, however, smoothing requires capturing significantly more images to reach an adequate $\mathrm{S} / \mathrm{N}$ ratio than OITM, which needs just two recordings, as shown in figure 5 . For high-contrast specimens, the noise level is nearly irrelevant compared to the signal level for detecting features, and neither smoothing nor OITM would lead to noteworthy improvements. Compared to bias noise, heat noise, and thermal noise, the level of photon noise is dominant in light microscopy. A contrast edge between two intensities $a$ and $b(a>b)$ can be detected if $a-\sqrt{a}>b+\sqrt{b}$. Although a bright uniform illumination can also increase the $\mathrm{S} / \mathrm{N}$, it may be avoided to prevent heat damage to the specimen. OITM does not only increase the contrast ratio $(a: b)$ of the signal, but it also decreases the photon noise level $(\sqrt{a}$ and $\sqrt{b})$ by adapting the illumination intensity regionally.

An increased $\mathrm{S} / \mathrm{N}$ ratio is beneficial to image analysis in digital microscopy, as the thresholding examples in figures 5 and 7 show. For automatic thresholding, we used the usual method by Otsu [23], which chooses a threshold to minimize the intraclass variance of black and white pixels. In digital microscopy, more advanced

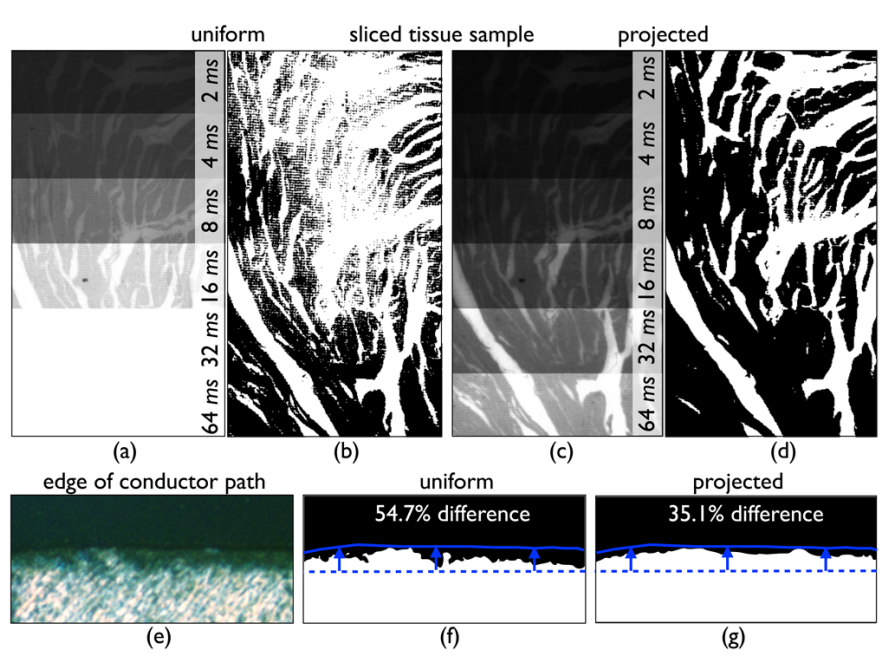

Fig. 7. Automatic thresholding [23] examples: HDR images were captured under uniform illumination and under contrast-stretched back-projected illumination $(\gamma=$ 1.0). Different exposures are shown for the sliced tissue sample in (a) and (c), while the results of automatic thresholding of the HDR images are presented in (b) and (d). Improvements by automatic thresholding for a lowcontrast edge of a conductor path (e) are also shown in (f) and $(\mathrm{g})$. The difference to the ground truth edge (solid line) is considered to be inversely proportional to the fill factor of the area between the solid line and the dashed line (compared to an entirely filled area in the best case).

scanning techniques can be applied to compose digital images with a high $\mathrm{S} / \mathrm{N}$ ratio by sampling the specimen with a relatively large series of structured illumination patterns. The advantage of our method is that an increased $\mathrm{S} / \mathrm{N}$ ratio can be achieved by capturing only two images. Although the quality of multi-sampling is likely to be higher, fast OITM can be beneficial in applications with real-time requirements.

\subsection{Enhancing Visibility of Spatial Structures}

Equation 3 stretches all entries within the selected luminance window to the displayable range of the projector (linearly or non-linearly, depending on the value of $\gamma$ ). Small contrast differences in bright or dark regions can be further amplified with a gamma compression $(\gamma<1)$ or a gamma expansion $(\gamma>1)$. This, however, can lead to extremely high local contrast values in the resulting optical image. While a stretched physical contrast can be beneficial in digital microscopy applications as long as the camera provides the required dynamic range, it is normally not advantageous when visibility is to be improved for direct observations through the oculars. The human visual system's dynamic range for simultaneous perception without re-adaptation is limited. Dark details 
neighboring bright ones are visually suppressed if their contrast is too high.

In scientific imaging, such as medical imaging, contrastlimited adaptive histogram equalization (CLAHE) [25], [30] is often applied to improve the visual detection of spatial structures in images. A histogram equalization spreads out the most frequent luminance values instead of stretching the entire luminance range exponentially. In the context of OITM, we investigated the efficiency of CLAHE for contrast-stretching the illumination pattern to enhance the visibility of spatial structures which are observed directly through the oculars. Furthermore, we coupled CLAHE with decorrelation stretch (DS) [2] to also maximize the difference between chrominances.
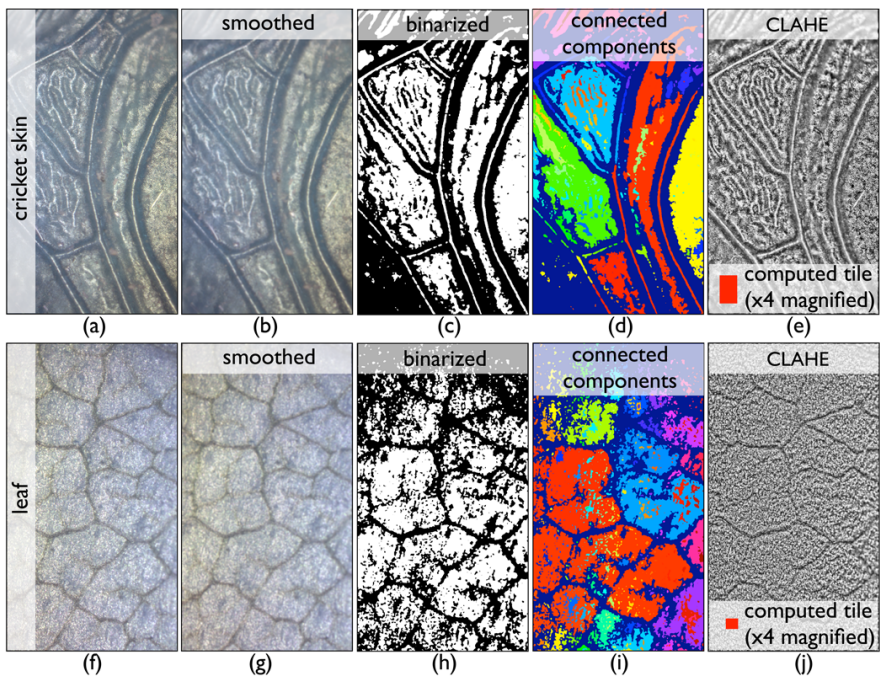

Fig. 8. Computing optimal tile size for CLAHE: First, $M$ $(a, f)$ is smoothed $(b, g)$ and binarized $(c, h)$. The average size of the bounding boxes of all connected components in the binarized image is used for $t_{x}$ and $t_{y}(\mathrm{~d}, \mathrm{i})$. The results of CLAHE on $M$ with the computed tile sizes (inlays are magnified $4 \mathrm{x}$ ) are presented in $(\mathrm{e}, \mathrm{j})$. These images were back-projected for OITM.

As before, all computations were carried out based on the white-light modulation image $M$. We computed CLAHE using the perceptually uniform luminance channel $\mathrm{L}^{*}$ of $M$ in the CIE $\mathrm{L}^{*} \mathrm{a}^{*} \mathrm{~b}^{*}$ color space. CLAHE examines the histogram of intensities for NxM subimages (called tiles of size $t_{x} \cdot t_{y}$ ) and calculates equalized mappings as lookup tables by cumulating the input histograms. These histograms are modified versions of the subimages' original histograms in which, at each intensity level, the contrast enhancement induced by the method is limited to a user-selectable maximum referred to as contrast limit $(c l)$. Once the mappings are calculated, neighbor subimage mappings are bilinearly interpolated to avoid boundary artifacts.

Thus, CLAHE requires setting three parameters: $c l, t_{x}$, and $t_{y}$. We derive the optimal initial setting for $c l$ from the pilot study in section 6 . We can, however, compute directly the optimal tile size $t_{x}, t_{y}$, which should match the size of the observed features and therefore depends on the specimen. CLAHE gives good results unless the difference between feature size and tile size is extreme, in which case excessively small tiles cause noise and excessively large tiles cause banding. Since specimens normally differ in feature size, we use the following steps to estimate a mean feature size (illustrated for two examples in figure 8):

We first apply a smoothing operator to reduce noise in $M$. Then we binarize the result and compute the connected components as described in [12]. The height and width of the average bounding box over all connected compounds is used for $t_{x}$ and $t_{y}$. Note that, since the average feature size throughout one specimen usually remains fairly constant, we can compute the tile size initially and keep it fixed for the specimen.

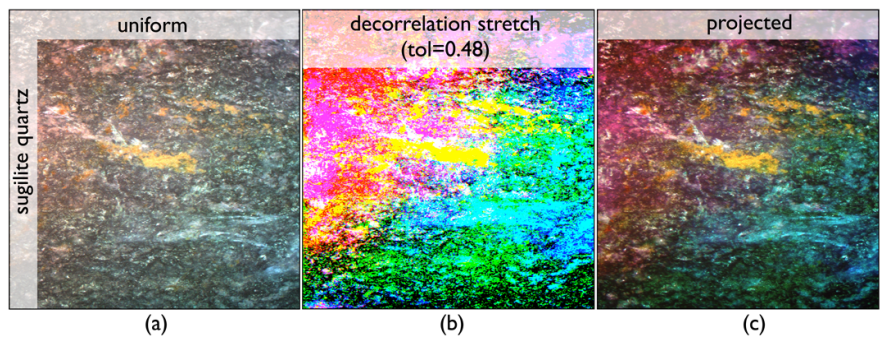

Fig. 9. Decorrelation stretch for increasing color contrast: In this example, a tolerance value of $t o l=0.48$ was chosen to enforce high RGB saturation. This maximizes the amplification of color contrast of the optical image (c) after applying DS to $M$ (a) and back-projecting the saturated result (b).

To enhance the color contrast while preserving the relative sense of hue, saturation, and intensity, we apply DS to the RGB channels of $M$, which includes removing the interchannel correlation found in $M$.

The derivation of the transformation matrix that decorrelates the RGB values of $M$ is explained in [3]. An additional linear contrast-stretch is applied by defining a tolerance parameter $(0 \leq t o l<0.5)$ that saturates the upper and lower RGB ranges (0...tol and 1-tol..1) in the final DS result. By modifying tol, the degree of saturation can be adjusted. Figure 9 illustrates an example.

Since CLAHE increases the luminance contrast and DS increases the color contrast, we combined both techniques as follows: We transformed the result of DS to the CIE L*a* $b^{*}$ color space, composed a new image by using $\mathrm{L}^{*}$ from CLAHE and $\mathrm{a}^{*}, \mathrm{~b}^{*}$ from DS, and finally converted the composition back to the RGB color space. This resulted in a contrast technique with a total of three adjustable parameters (while $t_{x}, t_{y}$ are computed): tol, $c l$, and an additional luminance scale factor $l$, which we apply to $L^{*}$ to increase and decrease the overall brightness. We compared the effects of these parameters on visual improvement and derived their optimal initial values in the course of a pilot study described in section 6. 


\subsection{Suppressing Highlights}

Besides low contrast, another issue that is particularly problematic for reflected illumination in optical microscopy are highlights on the specimen resulting from high-intensity surface- and subsurface reflections. As can be seen in the examples shown in figures 10 and 11, most details below or next to such highlights are lost under uniform illumination. This is disadvantageous not only for direct observations through the oculars, but also for image capturing and analysis in digital microscopy.
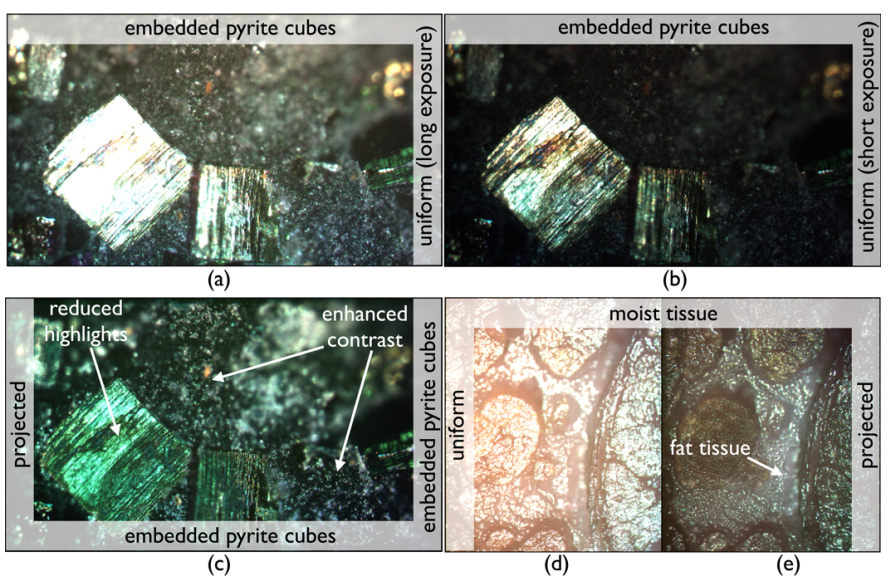

(e)

Fig. 10. Highlight clipping: Pyrite cubes embedded in rock under uniform illumination, captured at different exposures $(a, b)$ and under projected illumination that reduces bright reflections and enhances contrast in the background (c). Moist tissue under uniform illumination (d) and under projected illumination with suppressed highlights (e).

Given that the white-light modulation $M$ is known, such highlights can be suppressed effectively. For example, entries of $M$ that are above a high-intensity threshold can be set to a low-intensity magnitude. Since both values are specimen-dependent, they are user-defined. This reduces highlights by explicitly clipping the local illumination signal that causes high-intensity reflections. All entries in $M$ that are not clipped can still be contrast-enhanced as explained above. Figure 10 illustrates that this simple technique reveals details which are otherwise obscured by highlights under uniform illumination.

Projecting the complementary of $M$ (i.e., $1-M$ ) is yet another simple and efficient way of balancing contrast and consequently suppressing highlights. In contrast to highlight clipping, a suppression threshold and an attenuation magnitude need not to be defined manually, and attenuation transitions are smooth. As in highlight clipping, an additional contrast enhancement can be achieved by weighting a contrast-amplified version of $M$ with $(1-M)$.

Note that neither details below surface reflections nor details above or below subsurface reflections can be captured in HDR images or in low exposure LDR images under a uniform illumination, since they are superim-

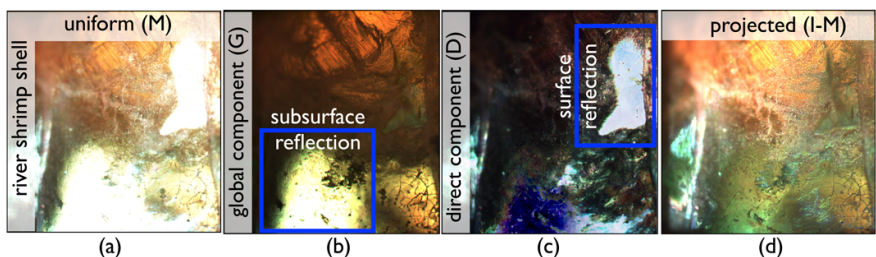

Fig. 11. Inverted illumination: River shrimp shell that reflects high light-intensities under uniform illumination (a). Separating global $(G)$ and direct $(D)$ illumination using the technique described in [19] reveals surface reflections and subsurface reflections (b,c). Projecting the complementary of $M$ (i.e., $1-M$ ) balances the contrast in the final image and reduces highlights caused by global and direct components at the same time (d). Note that $1-M$ is equivalent to $1-G-D$, and that we project only the luminance channel.

posed by the highlight signal. They can be revealed only by reducing the direct illumination regionally, as shown in figure 11.

More powerful segmentation methods can be applied to emphasize relevant foreground structures and to suppress irrelevant background structures. This serves not only to increase contrast and enhance visibility, since scattering is reduced in the suppressed background regions, but may also support optically augmented focus and context visualizations. As in contrast enhancement, suppression of highlights and background is fast with OITM because only one image must be captured to compute an optimized illumination pattern.

However, having to re-measure $M$ by recording the specimen under a uniform white illumination every time the specimen is moved or the microscope's focus or magnification are changed would disrupt the microscopy workflow. A continuous and uninterrupted handling is essential for many applications, including microscopic surgery. In the next section, we describe how the whitelight modulation can be estimated continuously while illuminating the specimen with an arbitrary pattern that is produced by one of the OITM techniques explained earlier. This makes possible implementing a projectorcamera closed-loop feedback that supports dynamic and interactive microscopy operations.

\section{Closed-Loop Feedback Illumination}

\subsection{Estimating White-Light Modulation}

We estimate the white-light modulation $M$ used for illumination computations in the next frame $(i+1)$ based on an element-wise division of the camera image $C$ captured at the current frame $(i)$ :

$$
M_{i+1}=C_{i} / \widehat{I}_{i},
$$

where $\widehat{I}$ is the corresponding illumination image that contains the estimated illumination power transferred to 
each color channel $(c)$ of the camera using a calibrated projector-camera transfer function $T^{p, c}$ :

$$
\widehat{I}_{i}^{c}=\sum_{p \in\{r, g, b\}}\left(T^{p, c}\left(I_{i}^{p}\right)-T^{p, c}(0)\right)+\frac{1}{3} \sum_{p \in\{r, g, b\}} T^{p, c}(0),
$$

where $p \in\{r, g, b\}$ and $c \in\{r, g, b\}$ are the projector and camera color channels respectively, and $I$ is the original illumination pattern in the projector's color space. $T^{p, c}$ is the illumination power transfer from projector color channel $p$ to camera color channel $c$, and $T^{p, c}(0)$ is the estimated projector black-level in the camera's color space. The calibration of $T^{p, c}$ is explained in section 5.2. Thus, $M$ estimates the specimen modulation with

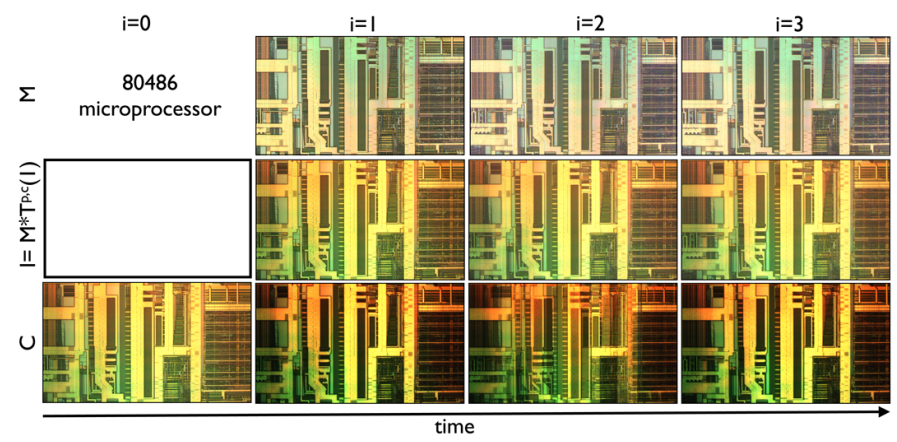

Fig. 12. Estimating white-light modulation: The illumination patterns $\left(I_{i}\right)$ and the corresponding camera images $\left(C_{i}\right)$ are the basis for estimating the specimen modulation $\left(M_{i+1}\right)$ under pure white light, which is used to compute the illumination pattern $\left(I_{i+1}\right)$ in the next frame. In this example, the illumination pattern is always the spectrum-scaled, estimated white-light modulation itself $\left(I_{i+1}=M_{i+1} \cdot T^{p, c}(1)\right)$. Hence, comparing each $I_{i+1}$ with the ground truth recording in $C_{0}$ becomes possible. In the ideal case, they are equal. The specimen was moved between frames 1 and 2 .

pure white light. We explain our prototypes that apply a three-panel LCD projector for pre-modulating the microscope's halogen lamp in section 5. This, however, does not produce a perfectly homogeneous spectrum. Nevertheless, we can compute the appearance of the estimated white-light modulation by multiplying $M$ with the projector's white light response in the camera's color space: $M \cdot T^{p, c}(1)$. Thus, a camera image captured under projected white light should have an appearance similar to the estimated white-light modulation scaled by the projector's spectrum in the camera's color space.

Figure 12 illustrates an example of four subsequent frames. The specimen was moved between frame 1 and frame 2. Despite the misregistered illumination in this frame, the white-light modulation was estimated correctly and the updated illumination pattern was registered automatically in the next frame. In this example, the spectrum-scaled estimated white-light modulation $\left(I=M \cdot T^{p, c}(1)\right)$ was back-projected, while $M$ represented the estimation under pure white light. Comparing the ground truth camera image captured under white projected light $\left(C_{0}=I_{1}\right)$ with the corresponding estimations $\left(I_{2}\right.$ and $\left.I_{3}\right)$ demonstrated that our method computes the white-light modulation well. It became apparent that the contrast of the estimations is slightly lower than in the captured image under physical white light projection (i.e., $C_{0}, I_{1}$ vs. $\left.I_{2}, I_{3}\right)$. The reason for this is that the optical images produced in back-projection exceed the dynamic range of the camera in a single shot $\left(C_{1 . .3}\right)$. This leads to a slightly underestimated contrast in $M$. We can counter this problem either by using a fast high dynamic range camera or by subsequently enhancing the contrast of $M$ as explained above.

\subsection{Handling Overestimation}

In principle, we can estimate the white-light modulation and update the illumination with a delay of only one frame, but at full frame rate. In practice, however, complex modulations that include interreflections, regional defocus due to the microscope's shallow depth-of-field, and movements of the specimen lead to problems that must be overcome.

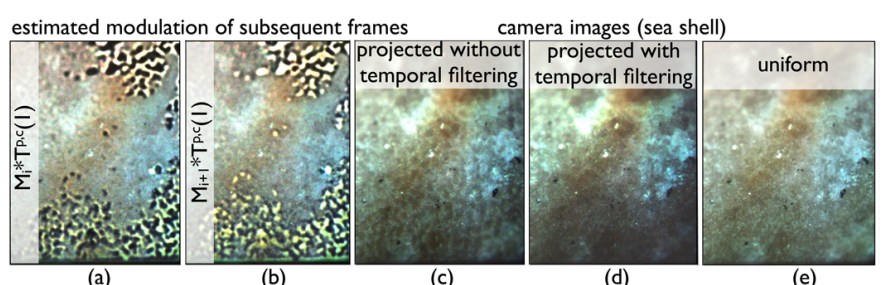

(a)

(b)

(c)

(d)

(e)

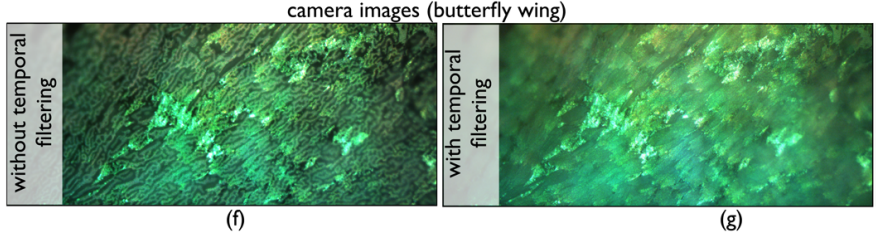

Fig. 13. Temporal filtering: When estimating white-light modulation while enhancing contrast, alternating artifacts appear where direct pixel correspondence between projector and camera is lost due to interreflections or defocus $(a, b)$. As they are contrast-enhanced continuously, their strength increases from frame to frame $(\mathrm{c}, \mathrm{f})$. Averaging two subsequent estimations results in stable images that are free of artifacts $(\mathrm{d}, \mathrm{g})$. Note that (d) and (g) appear more blurred than (c) and (f). The reason for this are the high-frequency artifacts in the unfiltered images. These artifacts are not part of the original images (compare (c,d) with (e), for example). The specimen in ( $\mathrm{g}$ ) is regionally defocused because of the microscope's limited depth-offield and not because of temporal filtering.

If a direct pixel correspondence between projector and camera is lost because of misregistrations caused by defocus or scattering, our closed-loop feedback estimation together with a contrast enhancement produces alternation artifacts, as illustrated in figure 13. The reason for this is that a single projector pixel that is defocused or scattered is captured by multiple camera pixels. These 


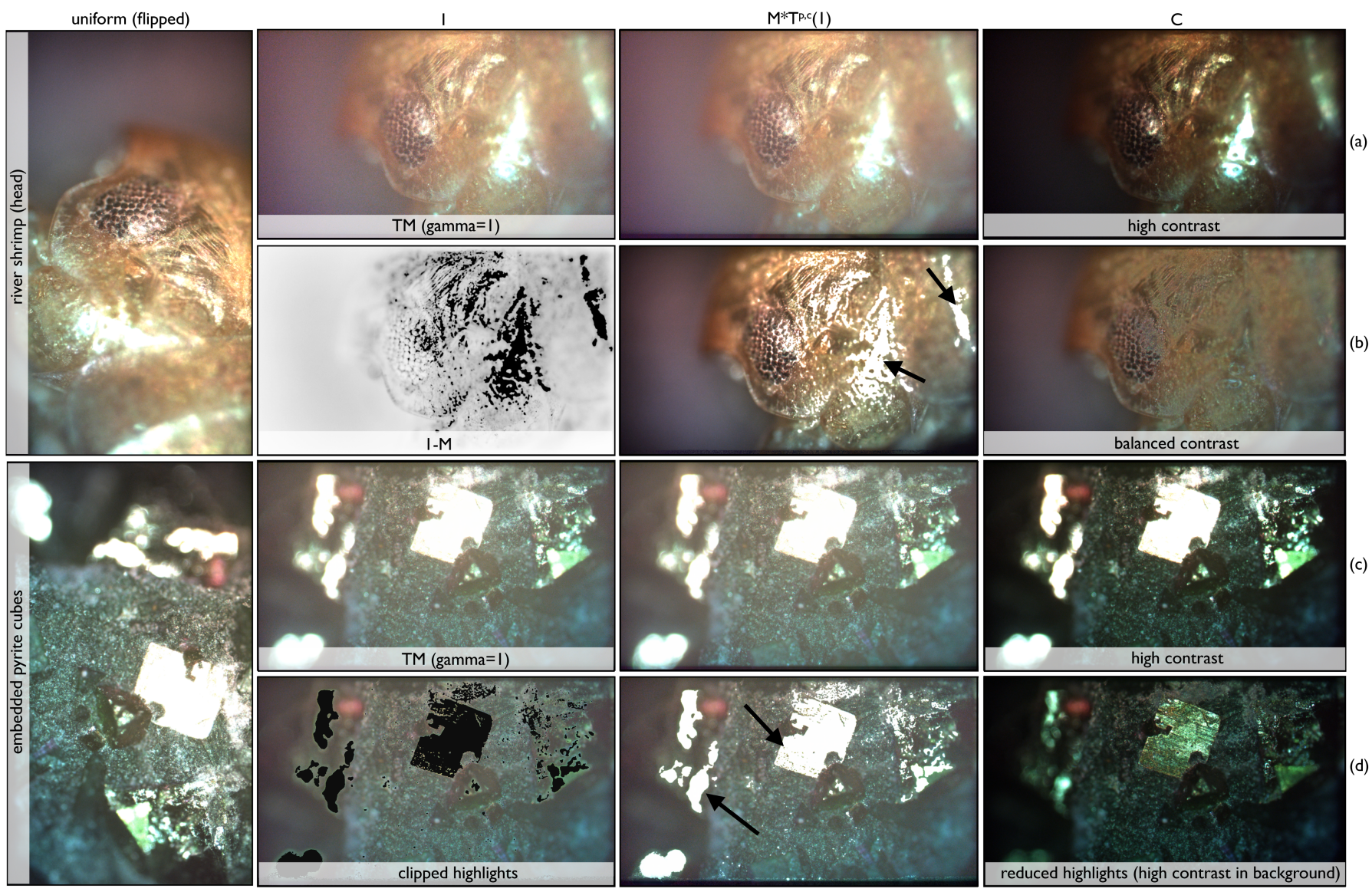

Fig. 14. Examples of our feedback loop for different illumination patterns $(I)$ : contrast enhancement using equation 3 and $\gamma=1.0$ (rows (a) and (c)). Note that in these cases the contrast of the optical images exceeds the dynamic range of the single-shot camera images $(C)$. This leads to a lower contrast in the estimated modulation $\left(M \cdot T^{p, c}(1)\right)$. Inverted illumination (row (b)) and highlight clipping (row (d)). Note that, since the contrast in $C$ is balanced in these cases, the contrast of the estimated modulation approaches the contrast of the corresponding images of the specimen when captured under uniform illumination. Reconstructed highlight regions (arrows) are clipped in the estimations.

camera pixels are in turn registered with other projector pixels. Thus, the direct relation between local irradiant illumination and corresponding local radiant reflectance that is assumed in equation 4 is invalid, which leads to an overestimation of white-light modulation values due to unknown indirect light transport. Consequently, the corresponding illumination values are updated wrongly, which influences the modulation estimation and the illumination computations in the next frame, and so on. The resulting artifacts alternate from frame to frame while their strength increases over time (because of contrast enhancement) until they become binary and eventually cover the entire field.

Unfortunately, we cannot separate the direct and indirect light-transport components of $M$ in one frame to overcome this problem. As explained in [19], multiple images must be captured under a structured illumination to measure the indirect component. This, however, is not feasible under an unobtrusive closed-loop feedback illumination. In classical control theory, solutions exist to regulate such underdamped situations. They cannot be applied in our case, since no reference output is given.
One positive characteristic of these artifacts is that their amplitude reverses in two subsequent frames, as can be seen in figure 13. Therefore, applying temporal filtering by averaging two subsequent modulations $\left(M_{i+1}^{\prime}=\right.$ $\left.\left(M_{i}+M_{i+1}\right) / 2\right)$ cancels the artifacts. Note that this way we do not increase the delay of our feedback-loop by more than one frame.

Movements of the specimen in between two frames, for instance, also lead to temporal misregistrations that can cause an overestimation of $M$ for regions that are illuminated at low intensities, as is the case in highlightclipped areas. If, for example, darkened areas in the illumination pattern that reduce highlights before a movement are projected onto non-highlight regions after a movement, we cannot provide a robust estimation of $M$ for these regions.

We overcome this problem as follows: If the estimated modulation is high for an entry in $M$, this suggests either a highlight at this position that must be compensated, or $M$ was overestimated due to the limitations explained above. We can distinguish these two cases by evaluating in addition the corresponding camera response. If it is 
below a certain threshold, the large modulation in $M$ is due to overestimation. This assumption is valid, since even the projector's black-level together with potential contributions from indirect illumination will lead to a camera response that is higher in highlight regions than in non-highlight regions. In this case, the invalid entries in $M$ are replaced by their nearest valid entries in $M$. The required threshold is computed automatically and updated for each current frame using the grayscale histogram computed for all camera pixels belonging to clipped highlight regions of the previous frame. The threshold is the intensity at the minimum that follows the first gradient decline in the histogram. This represents the lowest intensity reflected most by highlight regions only. Lower intensities are reflected by nonhighlight regions that are illuminated incorrectly, since they are misregistered briefly after movement. Figure 14 illustrates several feedback-loop examples with different illumination patterns that are computed for contrast enhancement, highlight clipping, and inverted illumination.

\section{Prototypes}

\subsection{Optical Configuration}

A Zeiss Axio Imager D1 served as a basic platform for all our experiments. This is a modular upright light microscope that offers the possibility of experimenting with different configurations (cf. figure 15). A Sony VPLAW 15 three-panel LCD projector was used for light modulation. Its original UHP lamp and its objective were replaced with the original microscope halogen lamp and new objective optics. The halogen lamp emits a more uniform spectrum than the UHP lamp, and the new objective optics (a combination of a Nikon $50 \mathrm{~mm}$ F1.2 digital camera objective and a Zeiss 44 M52x0,75 variable microscope camera adapter) match the pupils of projector and microscope imaging or illumination ports. We experimented with linearized Point Grey Flea 2 and Point Grey Dragonfly 2 CCD cameras attached to the top camera path deflection port. They were synchronized electronically with the projector to ensure frame-precise exposures.

For reflected illumination, the projector was attached to the infinite side camera path deflection port (cf. figure 15a). In this case, the light rays emitted by the projector and the light rays captured by the camera or observed through the oculars are parallel within the microscope optics, and are projected and imaged through the same microscope objective. Thus, projector, camera, and oculars share the same optical axis and focal plane. For transmitted illumination, the projector was attached to the microscope via the illumination port at the back normally used for conventional transmitted illumination (cf. figure 15b). The projector image must be focused using the condenser optics. Both the camera image and the image observed through the oculars must still be focused using the microscope objective.

\subsection{Calibration}

Projector and camera must be calibrated to measure their geometric and radiometric offsets. Even if both devices are optically co-aligned, different resolutions, chip/sensor sizes, and orientations around the optical axis can cause geometric misregistration. For reflected illumination, this is measured by projecting and capturing a Gray code on a surface mirror placed below the microscope objective. For transmitted illumination, the Gray code can be projected directly and captured in the empty field plane if projector and camera are focused at this plane.

The pixel correspondences were stored in a look-up table for real-time image warping during run time. The average misregistration of a projector pixel is currently one fifth of the size of a camera pixel. In addition, we measured the projector-camera transfer functions for all color channels and their combinations, which led to a total of nine transfer functions $\left(T^{p, c}\right)$ that indicate the illumination power transfer from projector color channel $p$ to camera color channel $c$. To this end, we projected all intensities of all color channels $p$ (onto the surface mirror for reflected illumination or directly at the field plane for transmitted illumination), measured the corresponding responses in all camera channels $c$, and stored them in look-up tables $\left(T^{p, c}\right)$. These look-up tables were used for real-time color correction during run time as explained in section 4.1. The exact implementation details of projector-camera calibration are beyond the scope of this discussion. Interested readers are referred to [7].

Furthermore, brightness and color inhomogeneity in the illumination were corrected by capturing one image $(W)$ under white illumination and one image $(D)$ under dark illumination (projector black-level) on a surface mirror. During run time, these images were used to apply a flatfield correction [16] to the projected illumination:

$$
I^{\prime}=m \frac{\left(I-T^{-1}(D)\right)}{\left(T^{-1}(W)-T^{-1}(D)\right)},
$$

where $I^{\prime}$ is the corrected and $I$ is the original illumination with mean pixel value $m$.

For reflected illumination and a 5-fold objective magnification, the projector pixel size on the field plane is $3.15 \mu \mathrm{m}$. The physical pixel size of the Point Grey Flea 2 camera is $1.1 \mu \mathrm{m}$ on the field plane. The composite camera pixel size (i.e., after de-mosaicing the Bayer pattern) is approximately $5.18 \mu \mathrm{m}$. The pixel sizes for the Point Grey Dragonfly 2 camera are $2.3 \mu m$ (physical) and approx. $7.05 \mu \mathrm{m}$ (composite). These sizes decrease with increasing objective magnifications. For transmitted illumination, the pixel footprint of the projector on the field plane is $1.64 \mu \mathrm{m}$.

The calibration remains constant if projector and camera share the same focal plane - no matter if the specimen is moved, the microscope re-focused, or its magnification changed. 


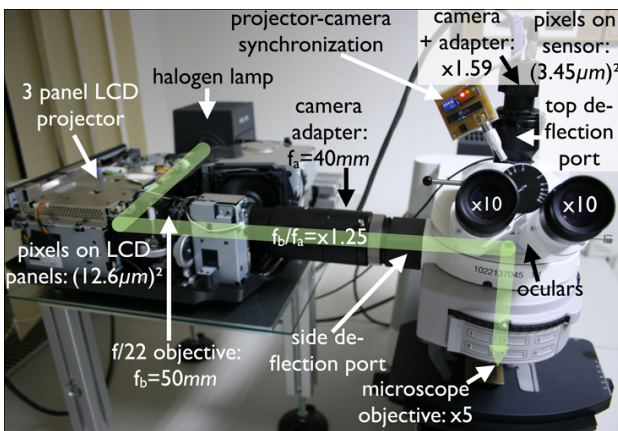

(a)

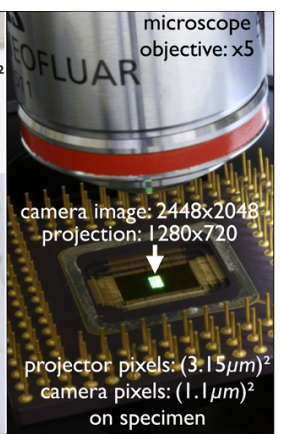

on specimen

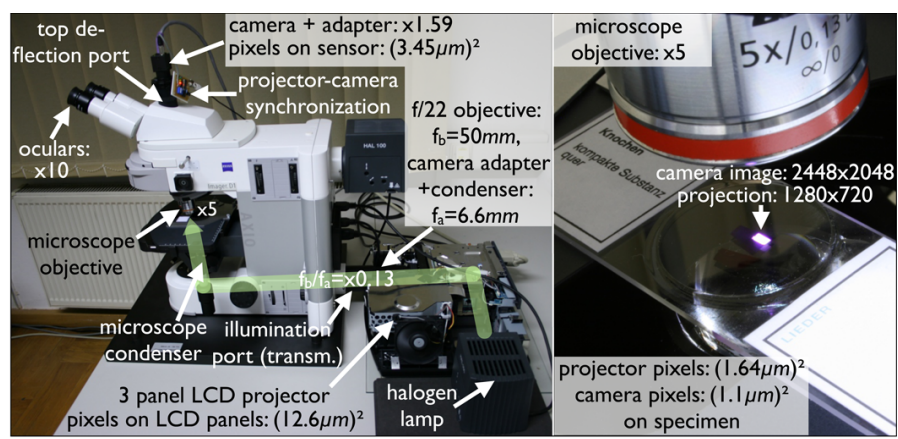

(b)

Fig. 15. Prototype configurations for reflected illumination (a) and for transmitted illumination (b). The numbers indicate the magnification factors of the individual components. The green arrows illustrate the illumination paths.

\section{Pilot Study}

\subsection{General Procedure}

Suppressing highlights or increasing the $\mathrm{S} / \mathrm{N}$ ratio using a controlled illumination leads to noticeable improvements. Thus far, however, it is still unclear if an enhancement of the visibility of spatial structures with OITM, as explained in section 3.3, is in fact beneficial in light microscopy. This is hard to predict, since we trade the higher contrast of a spatially modulated illumination for the increased brightness of a uniform illumination. An illumination pattern computed with the techniques described above always results in a lower adaptation luminance than a full uniform illumination.

To prove that OITM indeed leads to visibility improvements and to investigate the optimal initial parameterization of the contrast technique explained in section 3.3, we conducted a pilot study with a total of 41 subjects ( 30 male, 11 female, 21- 44 years of age with an average of 27 years). This study was carried out with the prototype configurations for reflected illumination.

After adapting to the brightness of the microscope, each subject was asked to observe three different specimens under four different lighting conditions. For two of these lighting conditions, we used OITM with the following contrast techniques applied to $M$ : CLAHE on the luminance channel only (no colors projected), and CLAHE+DS with an additional decorrelation of the chrominance, as explained in section 3.3. The remaining two lighting conditions were two uniform illuminations: one with the full possible brightness $(f u l l)$ and the other one with a brightness that leads to roughly the same adaptation luminance (adapt) as the previously observed OITM illumination. The different specimens are shown in figure 16: one with low contrast for evaluating OITM's impact on perceived contrast enhancement, one with color structures for evaluating OITM's impact on perceived color contrast enhancement, and one with low-reflectance for evaluating OITM's impact under low lighting conditions. The task for each subject consisted of two passes that were repeated for each of the three specimens.
TABLE 1

Specimen influence on parameters

$$
\left(F_{\text {critic }}=F_{(0.05,2,122)}\right) \text {. }
$$

\begin{tabular}{c|c|c|c}
\hline Parameter & $F$ & $F_{\text {critic }}$ & Influence \\
\hline \hline$c l$ (CLAHE) & 2.03 & 3.07 & no \\
$l$ (CLAHE) & 27.68 & 3.07 & yes \\
$t o l$ (CLAHE+DS) & 0.6 & 3.07 & no \\
$l$ (CLAHE+DS) & 29.37 & 3.07 & yes \\
\hline
\end{tabular}

\subsection{Contrast Parameter}

In the first pass, each subject was asked to adjust the contrast parameter for a specimen until the visibility of both small and large structures was optimal. This was repeated for each of the two contrast techniques. Thus, the $c l$ and $l$ parameters were adjusted first for CLAHE, and then $t o l$ and $l$ were adjusted for CLAHE+DS (while keeping $\mathrm{cl}$ from the previous adjustment). While looking through the oculars, a mouse was used to adjust the parameters in pairs, and its movements were mapped to $x, y$ changes. Note that it was always possible to readjust the original luminance of the illumination image by modifying the parameter $l$. For a given value of $l$ $(-255 \leq l \leq 255)$, the luminance was remapped to a range of $[l, 255]$ if $l>0$, or to a range of $[0,255+l]$ if $l \leq 0$.

After a Lilliefors test to validate that all our measurements were normally distributed, we conducted oneway ANOVA tests with the specimen as factor and the selected parameters as values to find out whether the observed specimen strongly influenced the parameter selection. We chose an alpha level of 0.05 . The results in table 1 show that only the adjusted luminance is influenced by the type of specimen. The contrast parameters $c l$ and $t o l$ are specimen-independent. To determine whether the contrast parameters are influenced by the adjusted luminance level, we performed unbalanced one-way ANOVA tests with four luminance intervals $([-255,-25],[-25,25],[25,75]$, and $[75,255])$ as factor and the contrast parameters as values. Again, an alpha level of 0.05 was chosen. The results in table 2 reveal that 
TABLE 2

Influence of luminance on parameters $\left(F_{\text {critic }}=F_{(0.05,2,40)}\right.$ for tol (low reflectance), $F_{\text {critic }}=F_{(0.05,3,40)}$ for all other parameters).

\begin{tabular}{c|c|c|c}
\hline Parameter & $F$ & $F_{\text {critic }}$ & Influence \\
\hline \hline$c l$ (low contrast) & 0.92 & 2.84 & no \\
$c l$ (colored structures) & 1.58 & 2.84 & no \\
$c l$ (low reflectance) & 0.26 & 2.84 & no \\
$t o l$ (low contrast) & 0.69 & 2.84 & no \\
$t o l$ (colored structures) & 0.82 & 2.84 & no \\
$t o l$ (low reflectance) & 1.28 & 3.23 & no \\
\hline
\end{tabular}

$c l$ and $t o l$ are also independent of the adjusted luminance level. Therefore, we chose the average of the medians from each sample (i.e., $c l=0.26$ and $t o l=0.25$ ) as initial parameters for the contrast technique explained in section 3.3. They can be fine-tuned any time during run time. The luminance value $l$ clearly depends on the reflectance of the specimen. For $l$, we also chose the average of the medians from each sample for both contrast techniques (i.e., $l=42.0$ for CLAHE and $l=49.33$ for CLAHE+DS) as initial values. However, we expect that, depending on the specimen, $l$ may require significant re-adjustment.

\subsection{Visibility Improvements}

After optimal adjustment of the parameters in the first pass, each subject was asked to compare the visibility of all four lighting conditions in the second pass. Thus, the subjects were initially able to switch freely between CLAHE and the two uniform illuminations (adapt was matched to the adaptation luminance produced by CLAHE). They were asked to rank the visibility of small and large structures for each of these illumination cases (with ordinal ranks from 1 to $3 ; 1$ =best). This was repeated for CLAHE+DS. Finally, the subjects could switch between CLAHE and CLAHE+DS for a direct ranking. Figure 16 illustrates the results averaged over all subjects.

Figure 16 shows that OITM always outperforms a uniform illumination - for all evaluated specimens and with respect to the visibility of small and large structures. While a higher adaptation luminance is generally better for uniform lighting conditions, a contrast-enhanced illumination improves visibility, even if its overall brightness is lower than that of a uniform illumination.

An enhancement of color contrast in addition to an enhancement of luminance contrast did not lead to significant perceptual improvements in our experiments, not even for specimens with colored structures. The reason for this might be that luminance contrast is the dominant factor in detecting structures. Since it did not change between CLAHE and CLAHE+DS, the detectability of structures remained unaffected. For small structures, a full uniform illumination outperformed CLAHE+DS. We think that the reason for this might be chromostereopsis: Colors with different wavelengths are refracted and
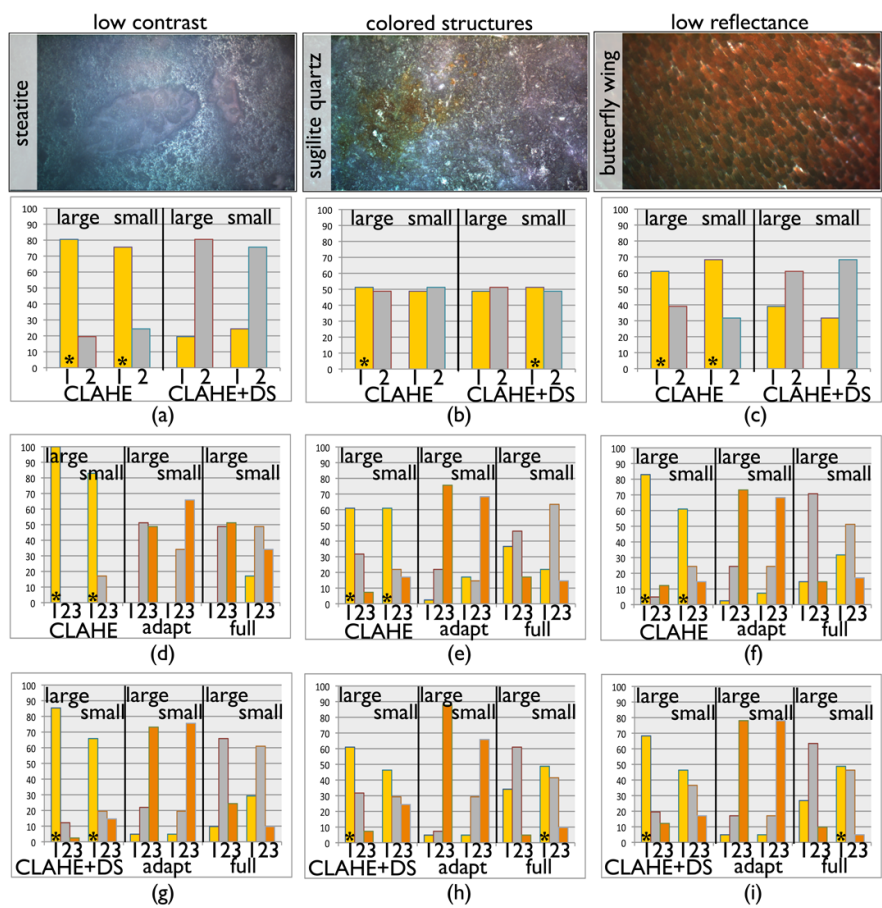

Fig. 16. Visibility improvements: Each diagram shows the ordinal ranking of the visibility of small and large structures ( $1=$ best), and the percentage averaged over the votes of all subjects. The first choices are indicated by an asterisk. The evaluated specimens are presented in the first row. The direct comparison of the two contrast techniques for each specimen is illustrated in the second row. The comparison between CLAHE and the two uniform lighting conditions is shown in the third row, and the comparison between CLAHE+DS and the two uniform lighting conditions is shown in the fourth row.

focused differently by the eye, which leads to visual defocus when strongly contrasting colors appear side by side. This is particularly problematic when resolving small features.

However, we believe that an enhancement in color contrast can improve the visual exploration of complex colored structures, since it provides a clearer segmentation between components. This was not evaluated in the course of our pilot study, because visual exploration tasks are usually more application-dependent and therefore less generalizable.

\section{Conclusion}

\subsection{Discussion}

For applications that require direct observation of the optical image through the oculars, OITM holds the potential to overcome the contrast constraints of human visual perception. For digital microscopy applications, the speed of OITM is probably one essential advantage over more advanced multi-exposure scanning and imaging techniques. In our current implementation, the entire closed-loop feedback computations together with 
contrast enhancement via non-linear tone-mapping (eqn. 3) and highlight clipping requires a total of $35.6 \mathrm{~ms}$ on a GeForce GTX 285. CLAHE and DS have not yet been ported to the GPU. They require $18 \mathrm{~ms}$ and 144 $m s$, respectively, on an Intel Core2Duo $3.0 \mathrm{GHz}$. All computations scale linearly $(O(N))$ with the resolution $(N)$ of the projector-camera system.

\subsection{Limitations}

Several limitations can be attributed to constraints of the components used: The dynamic range of the camera must be high for capturing and estimating the whitelight modulation of the specimen with adequate contrast. A high projector contrast and less scattering within the microscope optics will lead to additional improvements. Due to stray light remaining within the microscope optics, this contrast is as low as 105:1 in our current prototype.

When measured at the microscope's intermediate image plane, the objective resolving power is $2.24 \mu \mathrm{m}$ and the projector's pixel footprint is $31.5 \mu \mathrm{m}$ for reflected illumination and $16.4 \mu \mathrm{m}$ for transmitted illumination. Assuming a visual accuracy of the eye of $7.25 \mu \mathrm{m}$ on the intermediate image plane, the size of the projector pixels is currently above the resolving power of the human eye. This can be solved by using a higherresolution SLM or by optimizing the magnifications of oculars and objective. However, diffraction sets the limit at approximately 1000-fold magnification for the visible range in light microscopy. As a result, resolving features smaller than $0.2 \mu \mathrm{m}$ is not possible. This is also the lower limit of the SLM's pixel footprint in the visible range.

For transmitted illumination, the geometric and radiometric registration and the defocus of the projector and the camera are neither identical nor constant. The reason for this is that the camera image is captured through the microscope objective, while the projector image is displayed through the condenser optics. This means that the focus of the projector and the camera must be adjusted individually to the same field plane (i.e., the same plane of the specimen). Projector-camera calibration, as outlined above, must be carried out every time the microscope's parameters (focus and magnification) or the specimens are changed. A precise mechanical adjustment of the condenser with respect to the chosen magnification and focus settings would solve this problem. This, however, is currently not supported by our prototype.

The biggest limitation of our current prototype is that the peak luminance of the $100 \mathrm{~W}$ halogen illumination is reduced to $14 \%$ by the LCD panels and the additional optics of the projector. To support a better contrast sensitivity of high-frequency samples, we would need to employ a higher adaptation luminance. In addition, the exposure time of the camera must be high for samples with low reflection properties. Currently, this results in relatively slow update rates of 3-5 fps for our feedback loop, since camera exposures of up to $250 \mathrm{~ms}$ take a large share of the processing time. All of these problems can be addressed by optimizing the light throughput. Using a reflective instead of a transmissive SLM, such as a DMD, will certainly improve this situation significantly.

While the above drawbacks can be overcome by improving the microscope optics, the specimen itself sets general limitations. OITM becomes less efficient, the more the specimen scatters light.

\subsection{Future Work and Potential Applications}

We believe that an extension of real-time optical inverse tone-mapping to light-field microscopy [13], [14] can hold additional potential. Capturing, processing, and projecting a 4D light field instead of one single 2D slice of it (as done in our case) will open up further possibilities through the beneficial effect of a higher angular resolution in illumination and imaging. This may allow better results to be achieved for specimens with an excessive amount of scattering. The spatial resolution of lightfield microscopy, however, is currently too low for most applications in light microscopy. Thus far, we profit from the native resolving power of conventional microscopes, and our OITM implementation is limited only by the resolution of the camera and the SLM.

A possible alternative to our estimation of the specimen's modulation is to measure it continuously in wavelengths that are not used for illumination. The long wavelength of infrared (IR) light reduces the resolving power of a microscope and increases volume scattering significantly, and the short wavelength of ultraviolet (UV) light can destroy living cells and is therefore usually avoided. Moreover, color cannot be detected and processed with IR and UV light. Wavelength multiplexing in the visible range might be a better alternative that must be explored in future.

Further studies are required to investigate additional contrast techniques for optimal OITM illumination and their quantitative impact on contrast perception in more controlled user experiments. These techniques, however, might be specific to individual applications and specimens.

In general, we envisage benefits from OITM in a variety of fields that apply light microscopy and similar techniques. The contrast of tissue, for example, could be optimized in real time for microscopic surgery and endoscopy. In addition, specular highlights caused by liquids could be suppressed precisely. This may also be beneficial to other samples that cause specular highlights, such as crystals in mineralogy and conductor elements in electrical circuit inspections. In some cases, lowcontrast specimens may no longer require thin slicing or chemical processing (e.g., staining or decalcification as done for bones) to enhance contrast using a transmitted illumination technique, since OITM supports reflected illumination. For material science, OITM could be of advantage for real-time applications that process and observe low-contrast polymers, such as PVC. 


\section{ACKNOWLEDGMENTS}

We thank Anton Moffat and Enrico Geissler of Carl Zeiss AG Jena, and Sebastian Thiele and Ferry Häntsch of the Bauhaus-University Weimar for their support.

\section{REFERENCES}

[1] A.A. Adeyemi and N. Barakat and T.E. Darcie, Applications of digital micro-mirror devices to digital optical microscope dynamic range enhancement, Optics Express, vol. 17, no.3, pp.1831-1843, 2009.

[2] R.E. Alley, Decorrelation stretching as an aid to image interpretation, International Journal of Remote Sensing, vol. 8, pp. 12531254, 1987.

[3] R.E. Alley, Algorithm theoretical basis document for decorrelation stretch, Technical Report, Jet Propulsion Laboratory, Pasadena, CA, Ver. 2.2, August 15, 1996

[4] T. Amano and H. Kato, Real world dynamic appearance enhancement with procam feedback, Intl. Workshop on Projector-Camera Systems (poster), 2008.

[5] F. Banterle, P. Ledda, K. Debattista and A. Chalmers, Inverse tone mapping, Proc. Conference on Computer Graphics and Interactive Techniques in Australasia and Southeast Asia, pp. 349-356, 2006.

[6] O. Bimber and D. Iwai, Superimposing dynamic range, ACM Trans. Graph., vol.27, no.5, pp.1-8, 2008.

[7] M. Brown and A. Majumder and R. Yang, Camera-based calibration techniques for seamless multi-projector Displays, IEEE Trans. Visualization and Computer Graphics, vol.11, no.2, pp.193-206, 2005.

[8] P.E. Debevec and J. Malik, Recovering high dynamic range radiance maps from photographs, Proc. ACM Siggraph, pp. 369-378, 1997.

[9] A. F. Desimone and B. Crary, Spatial light modulator apparatus, Intl. Patent WO 03/040798 A1, May, 2003.

[10] K. Fujii, M.D. Grossberg and S.K. Nayar, A projector-camera system with real-time photometric adaptation for dynamic environments, Computer Vision and Pattern Recognition, IEEE Computer Society Conference on, volume 1, pp. 814-821, 2005.

[11] C. Gao, N. Ahuja and H. Hua, Active aperture control and sensor modulation for flexible imaging, IEEE International Conference on Computer Vision, pp. 1-8, 2007.

[12] R.M. Haralick, and L. G. Shapiro, Computer and robot vision, volume I, Addison-Wesley, pp. 28-48, 1992.

[13] M. Levoy, R. Ng, A. Adams, M. Footer and M. Horowitz, Light field microscopy, ACM Trans. Graph. vol. 25, no. 3 pp. 924-934, 2006.

[14] M. Levoy and Z. Zhang and I. McDowall, Recording and controlling the $4 \mathrm{D}$ light field in a microscope, Journal of Microscopy, volume 235, part 2, pp.144-162, 2009.

[15] H. Mannami, R. Sagawa, Y. Mukaigawa ,T. Echigo and Y Yagi, High dynamic range camera using reflective liquid crystal, IEEE International Conference on Computer Vision, pp. 1-8, 2007.

[16] D.B. Murphy, Fundamentals of light microscopy and electronic imaging, Wiley-Liss, ISBN0-471-25391-X, 2001.

[17] S.K. Nayar and V. Branzoi, Adaptive dynamic range imaging: optical control of pixel exposures over space and time, IEEE International Conference on Computer Vision, pp. 1168-1175, 2003.

[18] S.K. Nayar, V. Branzoi and T.E. Boult, Programmable imaging using a digital micromirror array, IEEE Computer Society Conference on Computer Vision and Pattern Recognition, pp. I-436I-443, 2004.

[19] S. K. Nayar, G. Krishnan, M. D. Grossberg and R. Raskar, Fast separation of direct and global components of a scene using high frequency illumination, ACM Trans. Graph., vol. 25, no.3, pp.935944, 2006.

[20] M.A.A. Neil, T. Wilson and R. Juskaitis, A wavefront generator for complex pupil function synthesis and point spread function engineering, Journal of Microscopy, vol.197, no.3, pp.219-223, 2000.

[21] M.V. Newberry, Signal-to-noise considerations for sky-subtracted CCD data, Pub. Astronomical Society of the Pacific, vol.103, pp.122-130, 1991.
[22] A.Y.M. Ng, C.W. See and M.G. Somekh, Quantitative optical microscope with enhanced resolution using a pixelated liquid crystal spatial light modulator, Journal of Microscopy, vol.214, no.3, pp.334-340, 2004.

[23] N. Otsu, A threshold selection method from gray-level histograms, IEEE Trans. Systems, Man, and Cybernetics, vol.9, no.1, pp.62-66, 1979.

[24] H. Park and M. -H. Lee and B. -K. Seo and H. -C. Shin and J.-I. Park, Radiometrically-compensated projection onto nonlambertian surface using multiple overlapping projectors, Proc. of Pacific-Rim Symposium on Image and Video Technology, pp.534544, 2006.

[25] S.M. Pizer, E.P. Amburn, J.D. Austin, R. Cromartie, A. Geselowitz, T. Greer, B.M. ter Haar Romeny, J.B. Zimmerman and K. Zuiderveld, Adaptive histogram equalization and its variations, Computer Vision, Graphics and Image Processing, vol. 39, pp. 355-368, 1987.

[26] E.C. Samson and C.M. Blanca, Dynamic contrast enhancement in widefield microscopy using projector-generated illumination patterns, New J. Phys., vol.9, no.10, pp.363-377, 2007.

[27] H. Seetzen, W. Heidrich, W. Stuerzlinger, G. Ward, L. Whitehead, M. Trentacoste, A. Ghosh and A. Vorozcovs, High dynamic range display systems, Proc. ACM Siggraph, pp.760-768, 2004.

[28] P.J. Verveer, Q.S. Hanley, P.W. Verbeek, L.J. van Vliet, and T.M. Jovin, Theory of confocal fluorescence imaging in the programable array microscope, Journal of Microscopy, vol. 189, pt. 3, pp. 192198, 1998.

[29] G. Wetzstein, D. Luebke and W. Heidrich, Optical image processing using light modulation displays, Computer Graphics Forum, in print.

[30] K. Zuiderveld, Contrast limited adaptive histogram equalization, Graphics Gems IV. chapter VIII.5, P.S. Heckbert (Eds.), Cambridge, MA, Academic Press, pp. 474-485, 1994. 